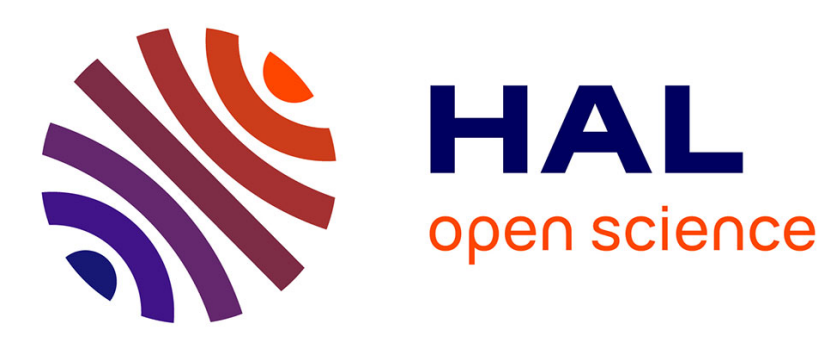

\title{
Tailoring Patterns of Surface-Attached Multiresponsive Polymer Networks
}

Benjamin Chollet, Loïc D'eramo, Ekkachai Martwong, Mengxing Li, Jennifer Macron, Thuy Quyen Mai, Patrick Tabeling, Yvette Tran

\section{- To cite this version:}

Benjamin Chollet, Loïc D'eramo, Ekkachai Martwong, Mengxing Li, Jennifer Macron, et al.. Tailoring Patterns of Surface-Attached Multiresponsive Polymer Networks. ACS Applied Materials \& Interfaces, 2016, 8 (37), pp.24870-24879. 10.1021/acsami.6b07189 . hal-01441498

\author{
HAL Id: hal-01441498 \\ https://hal.science/hal-01441498
}

Submitted on 31 Jan 2017

HAL is a multi-disciplinary open access archive for the deposit and dissemination of scientific research documents, whether they are published or not. The documents may come from teaching and research institutions in France or abroad, or from public or private research centers.
L'archive ouverte pluridisciplinaire HAL, est destinée au dépôt et à la diffusion de documents scientifiques de niveau recherche, publiés ou non, émanant des établissements d'enseignement et de recherche français ou étrangers, des laboratoires publics ou privés. 


\section{Tailoring Patterns of Surface-Attached}

\section{Multi-Responsive Polymer Networks}

Benjamin Chollet, ${ }^{1}$ Loïc D'Eramo, ${ }^{2}$ Ekkachai Martwong, ${ }^{1}$ Mengxing Li ${ }^{1}$

Jennifer Macron, ${ }^{1}$ Thuy Quyen Mai, ${ }^{1}$ Patrick Tabeling, ${ }^{2}$ Yvette Tran ${ }^{1} *$

${ }^{1}$ École Supérieure de Physique et de Chimie Industrielles (ESPCI Paris), PSL Research University, Sciences et Ingénierie de la Matière Molle, CNRS UMR 7615, 10 rue Vauquelin, F75231 Paris cedex 05, France. Sorbonne-Universités, UPMC Univ Paris 06, SIMM, 10 rue Vauquelin, F-75231 Paris cedex 05, France

${ }^{2}$ Institut Pierre-Gilles de Gennes (IPGG), 6-12 rue Jean Calvin, 75005 Paris, France

* Address correspondence to Yvette.Tran@espci.fr

KEYWORDS: polymer, hydrogel, responsive, thin film, grafting, pattern, photolithography 


\begin{abstract}
A new strategy for the fabrication of micro-patterns of surface-attached hydrogels with wellcontrolled chemistry is reported. The "grafting onto" approach is preferred to the "grafting from" approach. It consists in cross-linking and grafting preformed and functionalized polymer chains through thiol-ene click chemistry. The advantage is a very good control without adding initiators. A powerful consequence of thiol-ene click reaction by UV irradiation is the facile fabrication of micro-patterned hydrogel thin films by photolithography. It is achieved either with photomasks using common UV lamp or without photomasks by direct drawing owing to laser technology. Our versatile approach allows the fabrication of various chemical polymer networks on various solid substrates. It is demonstrated here with silicon wafers, glass and gold surfaces as substrates, and two responsive hydrogels, poly( $N$-isopropylacrylamide) for its responsiveness to temperature and poly(acrylic acid) for its $\mathrm{pH}$-sensitivity. We also demonstrate the fabrication of stable hydrogel multilayers (or stacked layers) in which each elementary layer height can widely range from a few nanometers to several micrometers, providing an additional degree of freedom to the internal architecture of hydrogel patterns. This facile route for the synthesis of micrometerresolute hydrogel patterns with tailored architecture and multi-responsive properties should have strong impact.
\end{abstract}




\section{INTRODUCTION}

Micro-patterned polymer hydrogels increase attention in biomedecine as cell culture substrates, permeable membranes, microvalves or microactuators. ${ }^{1-3}$ Hydrogels are very attractive owing to their biocompability and high deformability. Additional advantage of polymer hydrogels is their tunable properties through their chemistry. ${ }^{4}$ In particular, responsive hydrogels can undergo high volume changes under external stimuli such as temperature or $\mathrm{pH}^{5-6}$ For example, poly $(N-$ isoproprylacrylamide) (PNIPAM) hydrogel films show reversible and high thickness change with temperature by absorbing and expulsing water. ${ }^{7}$ The change could be four-fold or more depending on the crosslinks density of the hydrogel. ${ }^{8-14}$

Hydrogel micro-patterning can be performed using micro-molding and softlithography techniques. Soft lithography techniques are based on in situ polymerization of precursor monomer solutions against microstructure stamps (in general PDMS stamp) which are then removed by demolding. ${ }^{15-16}$ Kobel et al. proposed a different microfabrication technique called soft embossing to overcome the damage of soft and fragile polymer networks upon demolding. ${ }^{17}$ Alternatively, photolithography techniques enable hydrogel micro-patterning without the use of stamps. The photopolymerizable patches are obtained directly under UV light irradiation through photomasks. ${ }^{18}$ However, as radical polymerization is inhibited by the presence of oxygen, improved control on the chemical hydrogel formation can be performed by using preformed polymer chains instead of starting from monomers. Several groups have explored this photolithography approach with specific methodologies. For example, PNIPAM hydrogel patterns were immobilized on PTFE polymer substrate by low-pressure argon plasma treatment with a masking method. ${ }^{19}$ Using an alternate patterning technique, Chen et al. developed a grid pattern of two different nanothick microgels including poly(acrylic acid) pH-responsive 
microgel. ${ }^{20}$ Szilagyi et al. demonstrated that micrometer-scale microrelief with a high aspect ratio could be formed on a thin hydrogel layer composed of poly( $N$-isoproprylacrylamide $)$ and spirobenzopyran photoresponsive group. ${ }^{21}$

We propose a new approach for the micro-patterning of surface-grafted hydrogels with wellcontrolled chemistry, which has the advantages of simplicity and versatility. The method is inspired from the "grafting onto" technique: preformed ene-functionalized polymer chains are cross-linked and surface-attached by thiol-ene click reaction. It enables a better control than the approach starting from monomers. Additional advantage is that thiol-ene reaction can be performed under deep UV irradiation (around $260 \mathrm{~nm}$ ) without any addition of initiators. The proof-of-concept is achieved with responsive polymer hydrogels such as poly $(N$ isopropylacrylamide) for its temperature-responsiveness and poly(acrylic acid) for its $\mathrm{pH}$ sensitivity. But the extension to more general polymer networks can be envisaged depending on the properties targeted. The versatility of the method is illustrated with plane substrates such as silicon wafers, glass slides and gold surfaces. The thickness range of the hydrogel patterns is controlled by the precursor reactive polymer coating. Here, the spin-coating technique is chosen so that the thickness widely ranges from a few nanometers to several micrometers. The facile fabrication of micro-patterned hydrogels by photolithography is enabled with thiol-ene reaction as click chemistry. The versatility of our approach based on thiol-ene click chemistry owes to the commercial availability of many thiol molecules required for the cross-link reaction and the surface treatment. Bifunctional thiol molecules are used for the cross-linking and the treatment of gold surface and mercaptosilane for the functionalization of glass and silicon substrates.

In a previous publication, ${ }^{9}$ we reported the development of surface-attached hydrogel films with various internal architectures such as multilayers, interpenetrating networks and nanocomposite 
hydrogel films using this "grafting onto" approach based on thiol-ene click chemistry. In this present article, we focus on the method for the fabrication of micro-patterned hydrogels owing to thiol-ene reaction performed by UV irradiation. The parameters that control the formation of patterned hydrogels with multi-responsive properties are investigated. The photolithography can be performed either with photomasks using a common 8 Watt UV lamp at $254 \mathrm{~nm}$ or without photomasks by direct drawing using a $50 \mathrm{mWatt}$ laser at $266 \mathrm{~nm}$, which provides micrometerresolute patterns. We also show that these responsive hydrogel patterns can be integrated in microfluidic systems, demonstrating a new route for the facile fabrication of micro-actuators. 


\section{EXPERIMENTAL SECTION}

Thiol-modification of substrates. Prior to any chemical modification, silicon wafers and glass substrates are cleaned in a freshly prepared "piranha" solution (mixture of 70 vol\% of sulfuric acid and 30 vol\% of hydrogen peroxide $\left(35\right.$ vol\% in $\left.\mathrm{H}_{2} \mathrm{O}\right)$ heated at $\left.200^{\circ} \mathrm{C}\right)$. The solid substrates are then rinsed and sonicated in Milli-Q water and finally dried with nitrogen flow. Silanization with 3-mercaptopropyltrimethoxysilane is carried out on the freshly cleaned substrates to perform the thiol-modification of the surface. A solution of anhydrous toluene with 3 vol\% of mercaptopropyltrimethoxysilane (ABCR Gelest) is put in contact with the substrates for 3 hours inside a reactor under nitrogen. The solid substrates are rinsed and sonicated in toluene and finally dried with nitrogen flow. The thickness of the thiol layer on silicon wafer which is measured by ellipsometry, is $1 \mathrm{~nm}$ with a standard deviation lower than $10 \%$ of the average thickness.

Gold surfaces are made of a gold film (thickness is typically $100 \mathrm{~nm}$ ) evaporated on microscope glass slides. A chromium adhesion layer $(\sim 3 \mathrm{~nm})$ has to be first coated in order to improve adhesion of the gold film. Prior to metal deposition, the glass slides are cleaned in the "piranha" solution. Gold surfaces are modified with a thiol self-assembled monolayer. The substrates are immersed in a solution of dithioerythritol at $1 \mathrm{mM}$ in chloroform. They are kept immersed for 2 hours, in a sealed reactor to prevent evaporation of chloroform. The gold surfaces are then rinsed and sonicated in chloroform and finally dried with nitrogen flow.

Synthesis of hydrogel films. The synthesis of ene-reactive polymers was described in detail in our previous articles. ${ }^{8-9}$ Briefly, ene-functionalized polymers are synthesized in two steps for PNIPAM and one step for PAA. The two-steps method consists in free radical copolymerization 
of NIPAM and AA and ene-functionalization of the copopolymer by peptide reaction. First, poly(AA-co-NIPAM) copolymer is synthesized in water by free radical polymerization initiated by $\left(\mathrm{NH}_{4}\right)_{2} \mathrm{~S}_{2} \mathrm{O}_{8} / \mathrm{Na}_{2} \mathrm{~S}_{2} \mathrm{O}_{5}$ redox couple under nitrogen at room temperature. The amount of the $\left(\mathrm{NH}_{4}\right)_{2} \mathrm{~S}_{2} \mathrm{O}_{8} / \mathrm{Na}_{2} \mathrm{~S}_{2} \mathrm{O}_{5}$ initiators depends on the molecular weight of polymer chains targeted. For example, a molecular weight of $250 \mathrm{~kg} / \mathrm{mol}$ is obtained for the concentration of $\left(\mathrm{NH}_{4}\right)_{2} \mathrm{~S}_{2} \mathrm{O}_{8}$ and $\mathrm{Na}_{2} \mathrm{~S}_{2} \mathrm{O}_{5}$ in water equal to $10^{-3} \mathrm{~mol} / \mathrm{L}$. The reaction is allowed to proceed for 24 hours. The final solution is purified by dialysis in water. Second, the P(NIPAM-co-AA) copolymer is enefunctionalized by allylamine in water at room temperature in the presence of EDC/NHS couple. The reaction is allowed to proceed for 24 hours. The final solution is dialyzed in $\mathrm{NaCl}$ solution (to remove the unreacted EDC/NHS) and then in water. For ene-reactive PAA, only the step of funtionalization using allylamine is necessary.

Single-network films are prepared as follow. A solution containing ene-reactive copolymers and added dithioerythritol cross-linkers is dropped onto thiol-functionalized substrates for spincoating. The ratio of dithioerythritol to ene-reactive polymer units is 15 times. We chose to fix the conditions of spin-coating: the final angular velocity is $3000 \mathrm{rpm}$ and the spinning time is 30 seconds. For thermal activation of thiol-ene reaction, the polymer films are annealed at $120^{\circ} \mathrm{C}$ for 16 hours under vacuum. For deep UV irradiation, either a common 8 Watt UV lamp $(\lambda=254$ $\mathrm{nm})$ with photomasks or a $50 \mathrm{mWatt}$ laser $(\lambda=266 \mathrm{~nm})$ is used.

Multilayers films (or stacked polymer network layers) are fabricated by successive coating of a new layer over former layer, the thickness of each layer being decided by the molecular weight of the polymer and the concentration of the solution for coating. The very first layer is synthesized like a single-network film. The next layers are made with the same process: polymer films are coated and cross-linked by thiol-ene reaction. For thermal process, the multilayers 
architecture is obtained after 16 hours annealing at $120^{\circ} \mathrm{C}$ as for single-network films. The schematic of synthesis of surface-attached hydrogel films, single network and multilayers, is shown.

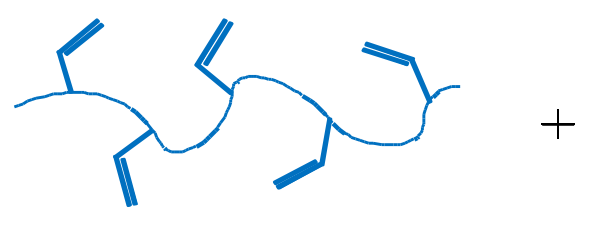

Ene-functionalized Polymer

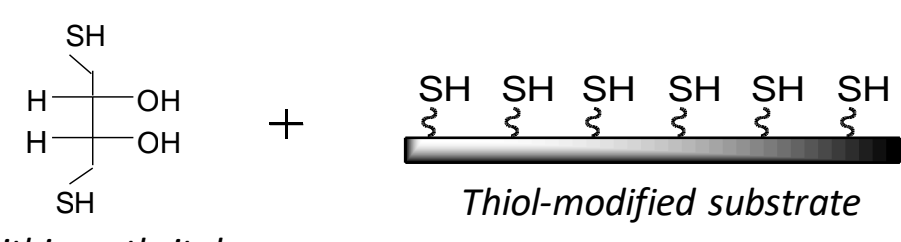

Dithioerythritol
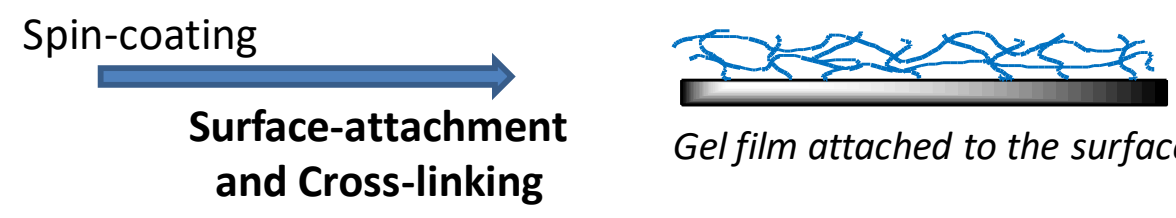

Gel film attached to the surface
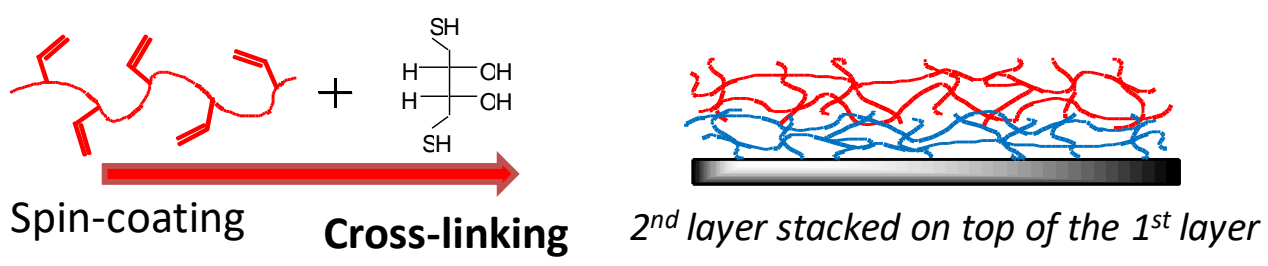

Patterns of surface-attached hydrogels. Hydrogel patterns are achieved under UV light irradiation through photomask using a (8 Watt) common fluorescent lamp $(\lambda=254 \mathrm{~nm})$. The photomask is aligned over the coated polymer film for typically 2 hours irradiation. Photomasks are obtained by patterning a $100 \mathrm{~nm}$-thick layer of chromium on quartz plates as quartz is transparent to UV contrarily to the chromium layer. The chromium layer is chemically wetetched with the use of a photoresist masking material (DOW Chemical). Another way to obtain hydrogel patterns is to perform the UV irradiation with a laser spot (diameter of $2 \mu \mathrm{m}$ or $20 \mu \mathrm{m}$ ) focused on the polymer film. The laser spot is moved over the surface for the direct drawing of highly resolute patterns. This laser lithography is performed using a KLOE DILASE 650 device 
(equipped with a $266 \mathrm{~nm}$ laser light source from CryLas $\mathrm{GmbH}$ ). Depending on the laser spot size, this option provides an output power of $3 \mathrm{~mW}$ and $5 \mathrm{~mW}$ for the $2 \mu \mathrm{m}$ and $20 \mu \mathrm{m}$ spot size respectively.

Surface characterization techniques. The thickness of hydrogel films is measured with spectroscopic ellipsometer Nanofilm EP3 (Accurion GmbH, Germany). For the measurements at silicon-air, we use the model with two layers. The first layer comprises silica and silane $(n=$ 1.46) which thickness was determined before grafting the hydrogel film. The second layer is the hydrogel film which thickness $h_{a}$ is measured while the refractive index depends on the polymer used to make the gel film. The refractive index of PNIPAM (respectively PAA) is 1.52 (respectively 1.53). Hydrogel film refractive index is 1.50 as it contains about $10 \%$ of water in air (water content lower than $10 \%$ was measured for humidity ratio between $20 \%$ and $60 \%$ ). The water content is weak so that the thickness of PNIPAM gel film in air can be considered as the thickness of dry film. Measurements in water (in situ) are performed with a temperaturecontrollable liquid cell equipped with thin glass walls (fixed perpendicularly to the light path with the angle of incidence at $60^{\circ}$ ). The polymer hydrogel film is modeled as a single layer with the thickness $h_{w}$ and a constant refractive index between that of water (1.33) and of the polymer. Since the hydrogel film is covalently attached to the substrate, The polymer amount is the same when immersed in water as the polymer is chemically grafted to the substrate. So we have: $n_{w}=(1.50-1.33) \times \phi_{p}^{w}+1.33$ and $h_{w} \times \phi_{p}^{w}=h_{a}$ with $\phi_{p}^{w}$ the volume fraction of polymer in water. The swollen thickness $h_{w}$, the refractive index of the film in water $n_{w}$ and the dry thickness $h_{a}$ are deduced from the fitting of the experimental data. The two equations should provide the same value of $\phi_{p}^{w}$ to ensure that the fitting is reliable. 
For Fourier transform-IR attenuated-total-reflection (FTIR-ATR) spectroscopy measurements, silicon substrates are used as infrared wavelength guides so that they are cut in special shape (70 $\mathrm{mm} \times 10 \mathrm{~mm} \times 1.5 \mathrm{~mm}$ trapezoidal crystal with an angle of $45^{\circ}$ ). The FTIR-ATR spectra are obtained using a Magna IR 550 (Nicolet) apparatus with a mercury-cadmium-telluride (MCT) detector cooled with liquid nitrogen. The spectra are recorded with a resolution of $2 \mathrm{~cm}^{-1}$ and a 256-scan accumulation.

The 3D surface profile of hydrogel patterns are measured with a noncontact optical profiler Wyco NT 9100 employing white-light interferometry. The measurements were analyzed and the profiles are plotted using Vision for Profilers software (Veeco Instruments).

Microfluidic experiments. Microfluidic channels are printed in NOA (Norland Optical Adhesive, photocurable monomer) according to the microfluidic stickers method. ${ }^{22} \mathrm{~A}$ drop of NOA 81 is deposited on a glass slide. A PDMS stamp is gently pressed onto the drop to create the channel structure. The NOA 81 is then insolated through the transparent PDMS stamp. The stamp is removed, a thin uncured adhesive layer remains thanks to the PDMS gas permeability. Putting the sticker onto contact with the substrate containing the surface-attached hydrogel patterns seals the microfluidic chip. A second insolation guarantees the adhesion between the sticker and the substrate by curing the adhesive layer. For fluorescence imaging, pictures are taken using a Leica DMI 6000B inverted microscope equipped with a 5X dry objective, coupled with a sensitive Hamamatsu ORCA-ER CCD camera. To control the temperature on the microfluidic device, a Linkam Scientific PE120 Peltier stage is inserted in the microscope XY stage. For better thermal conductivity, thermal grease (Radiospares) is applied in between the heating/cooling area and the glass slide of the microfluidic device. Channels are filled with a 0.8 $\mathrm{mM}$ solution of fluorescein (Sigma-Aldrich) in Milli-Q water. 


\section{RESULTS AND DISCUSSION}

\section{Synthesis of surface-attached hydrogel films}

The synthesis of surface-attached hydrogels is achieved with an inspired "grafting onto" strategy. It consists in cross-linking and grafting preformed ene-reactive polymers by thiol-ene click chemistry. The ene-functionalized polymer is coated on thiol-modified substrate with dithiol cross-linkers, the thiol-ene click reaction allowing simultaneously the cross-linking of chains and their covalent grafting to the substrate. It is the same process for the fabrication of multilayers films in which single-network films are stacked as for polymer layer-by-layer assemblies.

As shown in our previous articles, ${ }^{8-9}$ the variation of the thickness of surface-attached polymer network films can be achieved with the change of the viscosity of polymer solution for coating. Films of different thickness are then achieved by changing the viscosity with the variation of the polymer molecular weight and the polymer concentration. Figure 1 clearly demonstrates that surface-attached hydrogel films can be obtained with thickness ranging from a few nanometers to several micrometers using polymer concentrations from $1 \%$ to $10 \%$ and molecular weights from $70 \mathrm{~kg} / \mathrm{mol}$ to $700 \mathrm{~kg} / \mathrm{mol}$. The low molecular weights provide submicrometer thin layers with high precision and the high molecular weights allow the fabrication of micrometer thick films.

Figure 1 also displays the dry thickness of the hydrogel films as function of the number of coated/cross-linked layers. The linear increase of the thickness with the number of layers is demonstrated for two thicknesses of the elementary layer: $15 \mathrm{~nm}$ and $65 \mathrm{~nm}$. Each elementary 
thickness is controlled by the concentration or the molecular weight of the polymer solution for spin-coating . Any polymer hydrogel, PNIPAM or PAA, can be introduced in the multilayers, providing a degree of freedom to the internal architecture. It provides evidence of the simplicity and versatility of the approach to build stable polymer films with multi-responsive properties. Compared to usual layer-by-layer polymer assemblies in which the elementary adsorbed layer is a few nanometers-thick, the advantage of multilayers hydrogel films is the chance to vary the thickness at will in addition to change the nature of polymer network. All hydrogel films are stable and durable due to covalent bonds between chains and with the surface. If there is no chemical (or covalent) cross-linking, the hydrogel films will not be stable and polymer (free) chains will be removed during samples washing. For multilayers hydrogel films, entanglements between peripheral chains of two adjacent layers probably allow the stability. It can also be attributable to chemical (thiol-ene) bonds between these peripheral chains.
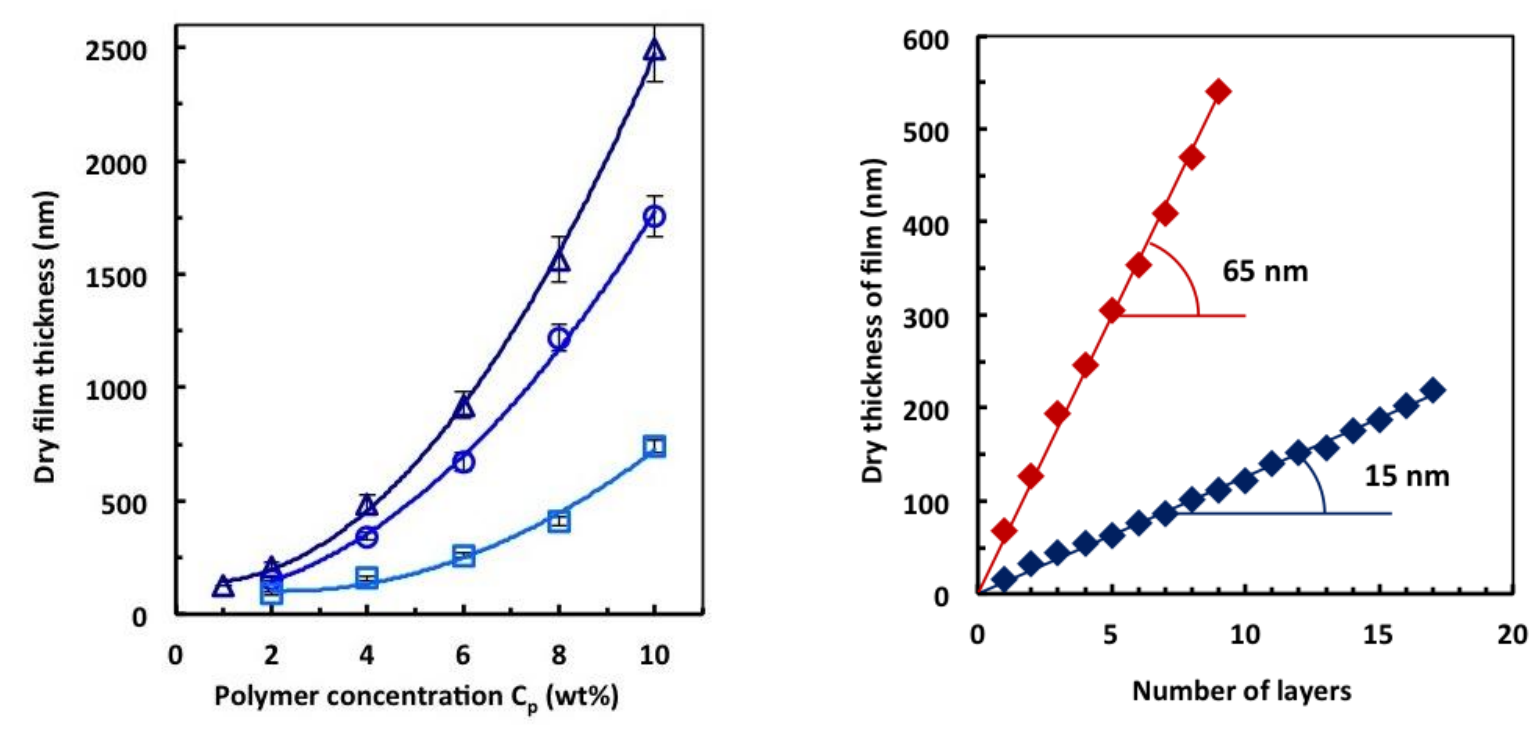

Figure 1. (Left) Dry thickness of PNIPAM hydrogel films as function of polymer concentration in the solution for spin-coating. The data are shown for various molecular weights (66 kg/mol: 
squared markers, $254 \mathrm{~kg} / \mathrm{mol}$ : round markers, $669 \mathrm{~kg} / \mathrm{mol}$ : triangular markers, the solid lines being guides for the eye). (Right) Dry thickness of hydrogel film as function of the number of layers. The linear increase is shown for two thicknesses of the single-network layer with 15 nm/layer and $65 \mathrm{~nm} / \mathrm{layer}$.

\section{UV irradiation for polymer cross-linking}
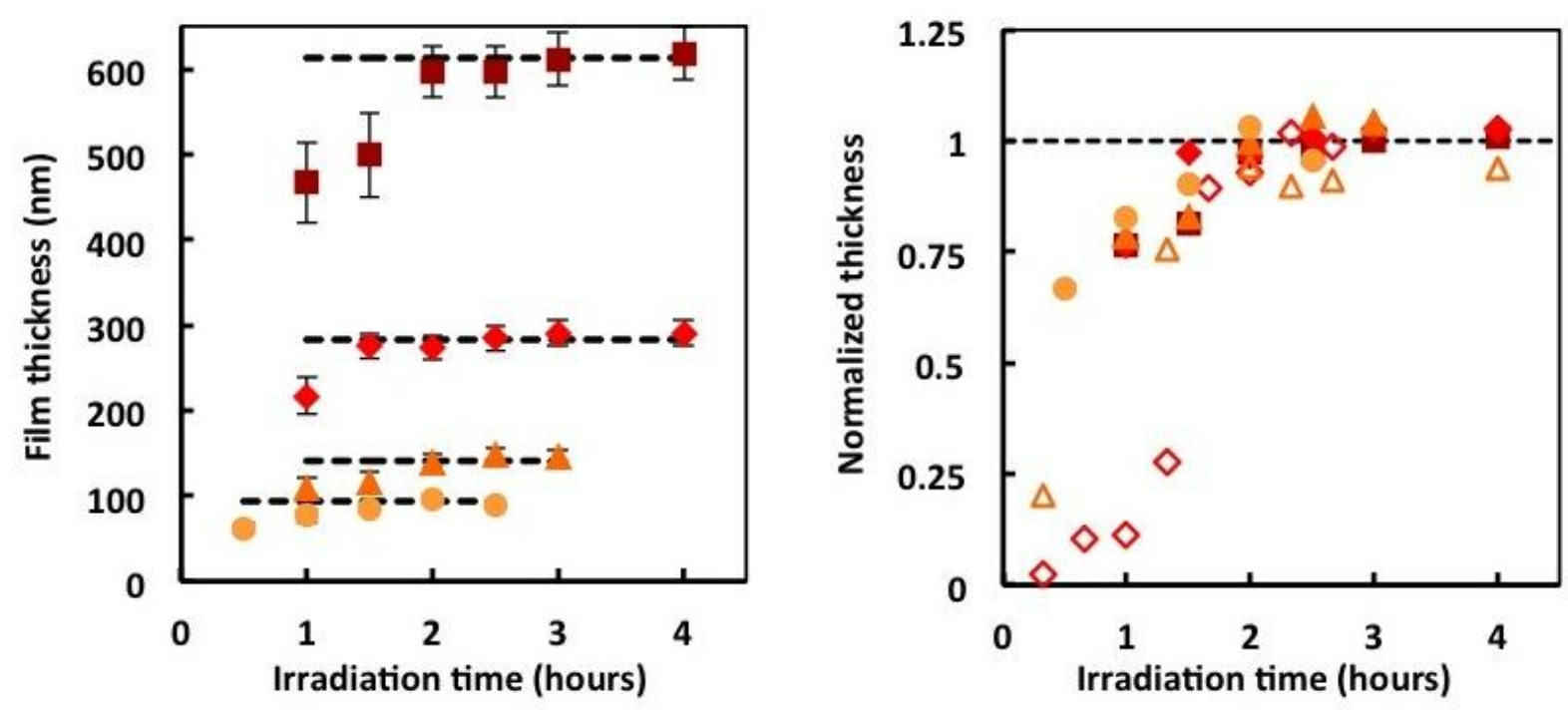

Figure 2. (Left) Dry thickness of PNIPAM hydrogel films as function of UV irradiation time. The data are shown for various concentrations of polymer in the solution for spin-coating (5 wt\%: brown square, 3 wt \%: red diamond, 2 wt\%: orange triangle, 1 wt $\%$ : yellow circle). The dotted lines indicate the maximum thickness of hydrogel films (obtained by heating activation). (Right) Normalized thickness of the hydrogel film as function of UV irradiation time for PAA (empty markers) and PNIPAM (filled markers) at different polymer concentrations in the spin-coating solution. The molecular weight of PNIPAM and PAA is $250 \mathrm{~kg} / \mathrm{mol}$. 
Surface-attached hydrogel films are obtained either by UV irradiation or thermal heating. Thiolene reaction can be achieved by selective deep UV irradiation without any added initiator for simultaneous cross-linking and surface-grafting of polymer chains. Figure 2 displays the film thickness as function of the UV irradiation time. The data are obtained with an irradiance of 2.4 $\mathrm{mW} / \mathrm{cm}^{2}$ at a wavelength of $254 \mathrm{~nm}$. The data are shown for various polymer concentrations of the spin-coating solution. The maximum thickness reached for each polymer concentration is the same than that obtained by heating process (during 16 hours). The representation with the normalized thickness (to the maximum value) is also displayed. The film thickness increases with the exposure time and reaches the maximum after 2 hours. The increase of the film thickness during the first two hours UV irradiation can be explained by partial cross-linking of polymer chains (uncross-linked chains are washed).

The effect of long time UV irradiation on the formation of surface-attached hydrogel films is also investigated. Figure 3 shows the (final) dry thickness of PNIPAM network films as function of activation time by UV irradiation and thermal heating. The hydrogel films are made from the same spin-coated PNIPAM layer using polymer solution with the concentration of 3\% and the molecular weight of $250 \mathrm{~kg} / \mathrm{mol}$. The thickness of the polymer network slightly increases from $270 \mathrm{~nm}$ to $320 \mathrm{~nm}$ from 5 hours to 20 hours heating and the maximum is reached after 20 hours. The thickness increase can be explained by partial cross-linking of polymer chains. This assumption is confirmed by the swelling properties of hydrogel films as shown next. The variation of the dry thickness of PNIPAM network films with UV irradiation time is completely different. At long time irradiation after 6 hours, the hydrogel films are damaged, with no films beyond 12 hours UV irradiation. 


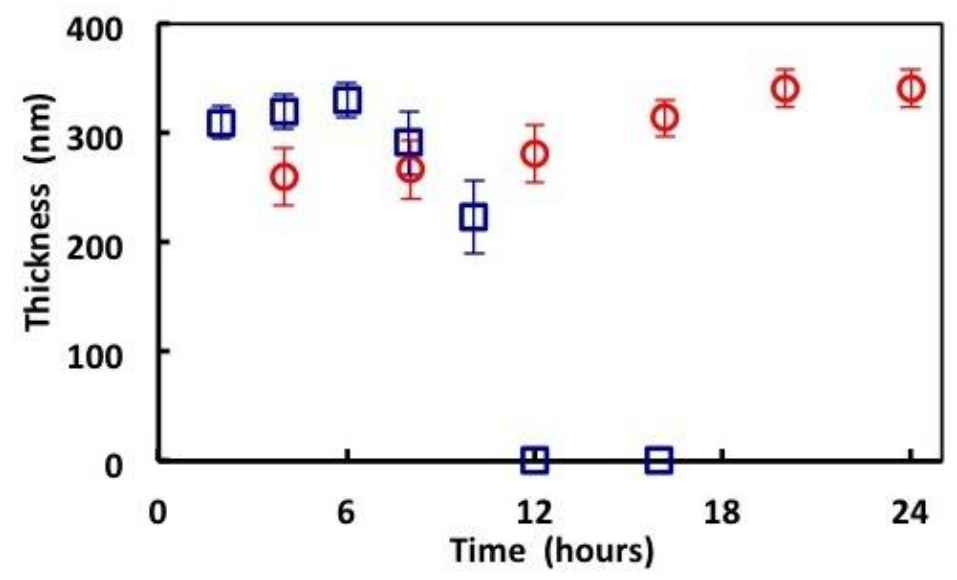

Figure 3. Final dry thickness of PNIPAM network film as function of activation time. The effect of UV irradiation (blue square markers) and thermal activation (red round markers) is compared. The hydrogel films are made from the same spin-coated polymer layer using polymer solution with the concentration of $3 \mathrm{wt} \%$ and the molecular weight of $250 \mathrm{~kg} / \mathrm{mol}$.

The determination of the thickness of hydrogel films in water is an additional and valuable means to finely control the synthesis of surface-attached hydrogel films as the swelling if hydrogel films increases with decreasing crosslinks density. Figure 4 shows the swelling ratio as function of activation time by thermal heating and UV irradiation. The swelling ratio is the ratio of the swollen thickness of hydrogel films (measured in water) to the dry thickness (measured in air). It is measured at two different temperatures. At $25^{\circ} \mathrm{C}$, below the LCST, PNIPAM hydrogel films are supposed to swell with high swelling ratio if the polymer network is less cross-linked. At $40^{\circ} \mathrm{C}$, above the LCST, PNIPAM hydrogel films are expected to collapse.

At short time heating, lower than 20 hours, the swelling ratio at $25^{\circ} \mathrm{C}$ decreases to reach the constant value of 3.2. It is likely due to the increase of the thiol-ene crosslinks reaction with time 
heating. At $40^{\circ} \mathrm{C}$, collapsed PNIPAM hydrogel films have the same swelling ratio of 1.5 , no matter the time heating. As discussed in our previous publications, water is not entirely expulsed from the PNIPAM collapsed hydrogel which contains about $30 \%$ of water. ${ }^{8-9}$ It was found for PNIPAM collapsed hydrogel films no matter the crosslinks density ${ }^{11-14}$ and also for PNIPAM collapsed brushes. ${ }^{23-24}$ The collapsed state does not depend on the architecture of PNIPAM film, network or brush. It is only decided by polymer-solvent interaction or $\chi$ Flory parameter.

The damage of hydrogel films with long time UV irradiation is supported by the variation of the swelling ratio at $40^{\circ} \mathrm{C}$. The swelling ratio found is not 1.5 for supposedly collapsed PNIPAM films as expected, but it increases improperly with temperature. It can be attributable to oxidation and accelerated ageing with UV irradiation. As a result, the optimized UV irradiation time using the 8 Watt lamp at $254 \mathrm{~nm}$ is between 2 hours and 6 hours. In this time range, the dry thickness and the swelling ratio of hydrogel films are invariable. 

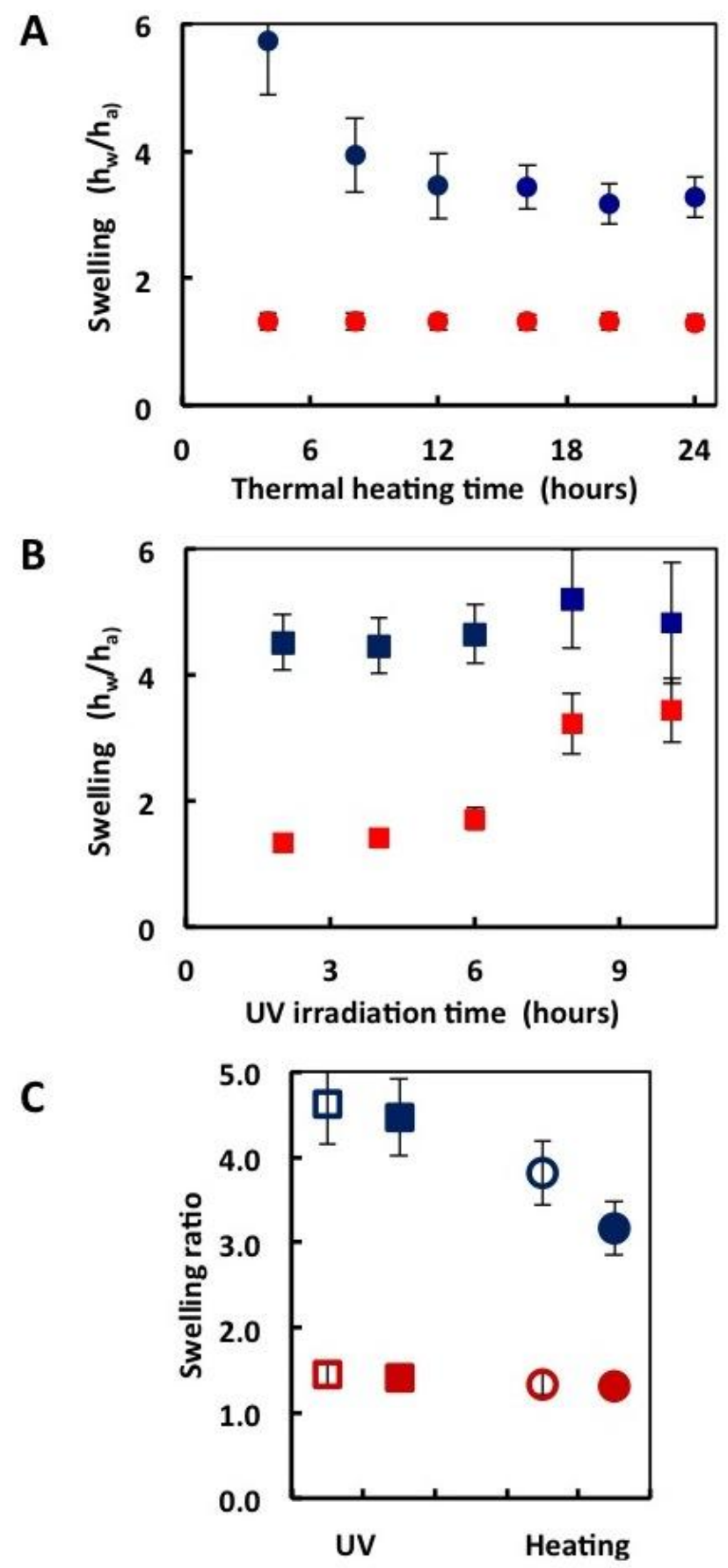

Figure 4. (A) and (B) Swelling ratio of PNIPAM hydrogel films as function of activation time. The swelling ratio $h_{w} / h_{a}$ is the ratio between the hydrogel thickness in water and the dry thickness in air. The swelling ratio is measured at $25^{\circ} \mathrm{C}$ below the LCST (blue) and at $40^{\circ} \mathrm{C}$ above the LCST (red). The effect of UV irradiation (square markers of Figure B) and thermal 
activation (round markers of Figure A) is compared. The dry thickness of these hydrogel films is shown in Figure 3. (C) Swelling ratio at $25^{\circ} \mathrm{C}$ (blue markers) and $40^{\circ} \mathrm{C}$ (red markers) of PNIPAM hydrogel films made from reactive polymers with two different ratios of enefunctionalization 2\% (open markers) and 3\% (filled markers) and with two thiol-ene crosslinking processes (UV irradiation and thermal activation).

Figure 4 also displays the swelling properties of PNIPAM hydrogel films obtained with UV irradiation and thermal activation of thiol-ene cross-linking reaction, the data being shown for hydrogel films made from reactive polymers with two different ratios of ene-functionalization ( $2 \%$ and $3 \%$ ). The swelling ratio of collapsed hydrogel films at $40^{\circ} \mathrm{C}$ is about 1.5 for any crosslinking activation process and any ratio of ene-functionalization. It is independent of the crosslinks density as discussed above. The swelling ratio of swollen films at $25^{\circ} \mathrm{C}$ decreases with the ratio of ene-reactive units. It is lower for thermal activation than for UV irradiation corresponding to higher yield of thiol-ene cross-linking reaction for thermal process. The values of swelling ratio are comparable to those obtained by Toomey et al. with benzophenone photocrosslinkers. They demonstrated that the swelling ratio is a power law of the proportion of benzophenone photo-crosslinkable units (from 0.5 to $14.3 \%$ ) with the exponent of $-1 / 3 .{ }^{12}$ Our values of swelling ratio of 4 (respectively 3 ) with $2 \%$ (respectively $3 \%$ ) ene-reactive polymers fit Toomey's results. Moreover, the comparison between the swollen and collapsed states shows phase transition with high amplitude (3 to 4). This high amplitude transition could be advantageously exploited for applications of hydrogel films using temperature-responsive properties. 


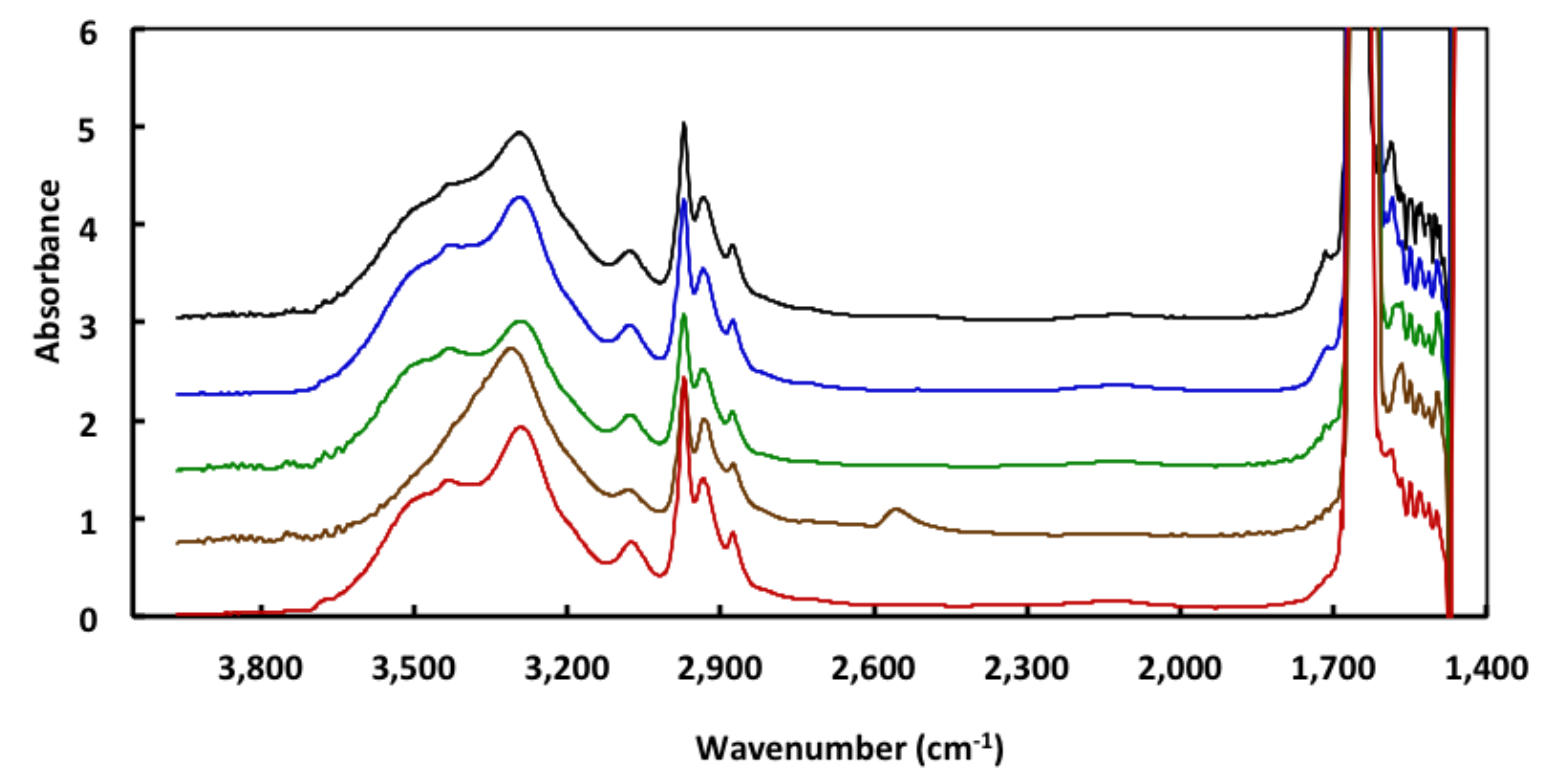

Figure 5. FTIR-ATR spectra of PNIPAM films. The absorption spectra are obtained with PNIPAM films at the surface of silicon prisms-waveguides: spin-coated film containing PNIPAM (red), spin-coated film containing PNIPAM and dithioerythritol (brown), PNIPAM hydrogel film after 24 hours thermal activation (green), PNIPAM hydrogel film after 2 hours UV irradiation (blue), PNIPAM hydrogel film after 10 hours UV irradiation (black).

Figure 5 shows FTIR-ATR spectra of PNIPAM films. Five absorption spectra are compared: the spin-coated film containing only PNIPAM, the spin-coated film containing PNIPAM and dithioerythritol before thiol-ene activation, PNIPAM hydrogel film after the grafting and crosslinking of chains by thiol-ene reaction either by thermal activation or by UV irradiation after 2 hours and 10 hours. 
As silicon is used as infrared waveguides, it is difficult to quantitatively analyze the wave number range below $1600 \mathrm{~cm}^{-1}$ because of the high absorbance of the silicon substrates $(\mathrm{O}-\mathrm{H}$, Si$\mathrm{O}$, Si-Si bonds) so that information can only be extracted from $4000-1600 \mathrm{~cm}^{-1}$ range. The absorption bands between 3100 and $2800 \mathrm{~cm}^{-1}$ are characteristic of asymmetric and symmetric stretching vibrations of $\mathrm{CH}, \mathrm{CH}_{2}$ and $\mathrm{CH}_{3}$ groups. The absorbance is expectedly the same for all spectra. The loss of the peak at $2500 \mathrm{~cm}^{-1}$ attributable to dithiol cross-linkers (S-H stretch) for spectra of PNIPAM films after grafting and cross-linking is explained by the thiol-ene reaction and the removal of dithiol in excess. It is also consistent with the high increase of the absorption band at $3300 \mathrm{~cm}^{-1}$ characteristics of $\mathrm{O}-\mathrm{H}$ (due to $\mathrm{H}$ bonds) found in the spectrum before crosslinking (because of the presence of dithioerythritol in excess), this broad and high band even hiding the peak at $3430 \mathrm{~cm}^{-1}$ characteristics of N-H amide groups. Unfortunately, it is not possible to quantify the thiol-ene reaction for two main reasons. First, the absorption peak characteristics of S-C bond is at very low wave number (around $700 \mathrm{~cm}^{-1}$ ). Second, the thiol-ene ratio is weak ( maximum at 2-3\%), ${ }^{1} \mathrm{H}$ NMR showing a ratio of ene- functionalization around 2$3 \%$.

The spectra of PNIPAM network films after 2 hours and 10 hours UV irradiation are the same while the alteration of temperature-responsive properties is observed at long time irradiation (see Figure4). The same absorbance of the peaks between 3600 and $2800 \mathrm{~cm}^{-1}$ (characteristic of N-H amide groups, $\mathrm{CH}, \mathrm{CH}_{2}$ and $\mathrm{CH}_{3}$ groups) is indicative of no major damage of PNIPAM chains. In fact, the accelerated oxidation of the polymer network film with UV irradiation, even not obviously confirmed by FTIR-ATR, seems to be the most reliable assumption. It can be supposed that the formation of hydrophilic sulphonate groups (around $1300 \mathrm{~cm}^{-1}$ and $1100 \mathrm{~cm}^{-1}$ ) 
in the PNIPAM hydrogel films which is favored by UV irradiation, is responsible of the cancelation of the collapse behavior of PNIPAM hydrogel films at $40^{\circ} \mathrm{C}$.

\section{Micro-patterns of polymer network}

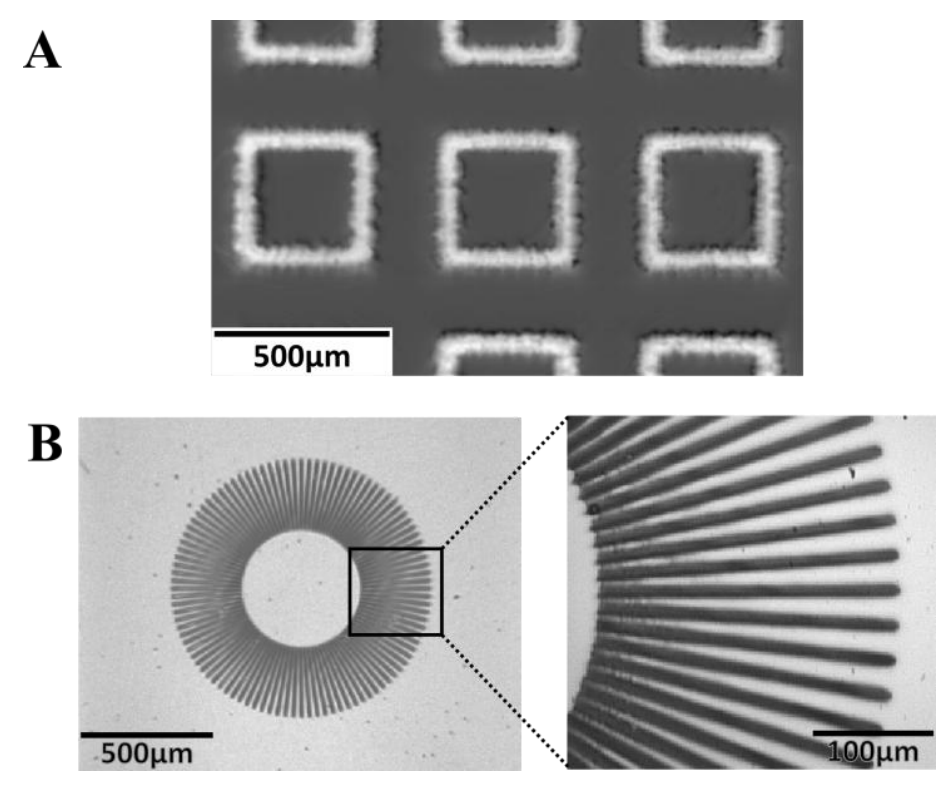

Figure 6. Optical microscopy images of PNIPAM hydrogel patterns. The patterns are obtained with a 8 Watt UV lamp through photomasks. Square patterns with $50 \mu$ m-wide and $2.5 \mu$-thick walls are grafted on glass surface (A). $6 \mu \mathrm{m}$-wide and $250 \mathrm{~nm}$-thick patterns are grafted on silicon wafer $(B)$.

The advantage of selective UV irradiation is the facility to fabricate hydrogel patterns. Figure 6 shows two examples of surface-attached hydrogel micro-patterns using a common 8 Watt UV 
lamp through photomasks, the patterning corresponding to a negative photolithography. 400 microns-wide squares with 50 microns-wide and 2.5 microns-thick walls are covalently grafted on glass susbtrate. The images of the patterns on the glass surface are observed using optical profilometer. The glass surface is dark grey and the hydrogel blocks appear in bright tones proportionally to the hydrogel thickness. Another example of photolithography using photomask is shown with 6 microns-wide and 250 nanometers-thick lines of hydrogels grafted on silicon wafer. The images on the silicon wafer are obtained with reflected light microscope. Here, the reflective silicon substrate is bright while the polymer patterns are in black tone.

A

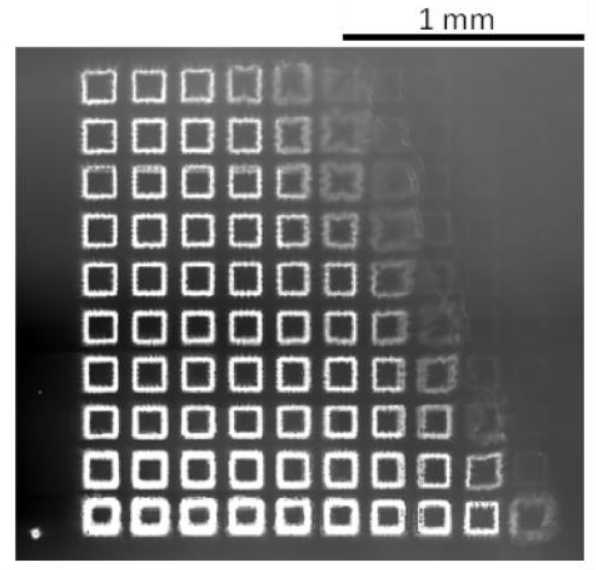

B

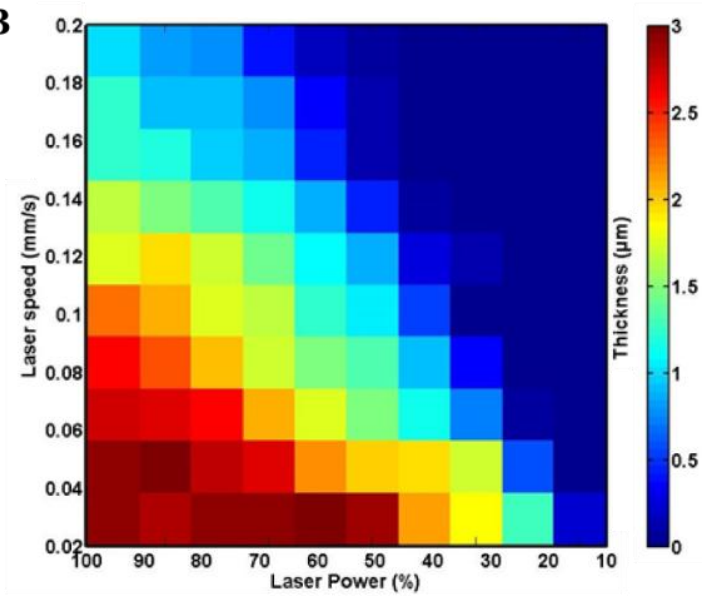

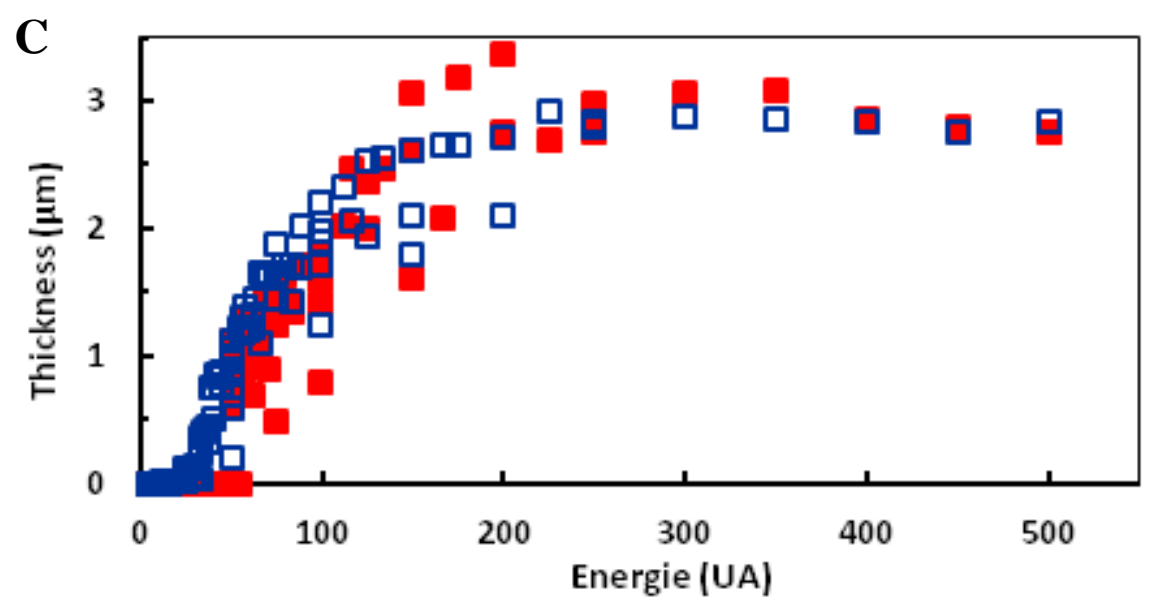


Figure 7. (A) Optical profilometer image of patterned PNIPAM hydrogel squares on glass substrate using $50 \mathrm{mWatt}$ laser at $266 \mathrm{~nm}$ (DILASE 650 device) with the spot of $20 \mu \mathrm{m}$ diameter. The substrate is dark and the hydrogel cavities appear bright. (B) Cartography of hydrogel thickness deduced from the optical 3D profile as function of two parameters of laser lithography: the laser speed and the laser power. The cartography is obtained using the laser spot of $20 \mu \mathrm{m}$ diameter: the laser speed ranges from 0.02 to $0.2 \mathrm{~mm} / \mathrm{s}$ and the corresponding thickness is up to $3 \mu \mathrm{m}$. (C) PNIPAM hydrogel thickness as function of UV irradiation energy using laser lithography. The data are shown for the laser spot of $20 \mu$ m diameter (blue open markers) and 2 um diameter (red filled markers).

The same patterns with square enclosures as in Figure 6 can also be achieved from direct writing (without photomask) using a $50 \mathrm{mWatt}$ laser at wavelength $(\lambda=266 \mathrm{~nm})$ integrated in the lithography device. Depending on the laser spot size, it provides an output power of $3 \mathrm{~mW}$ and 5 $\mathrm{mW}$ for a $2 \mu \mathrm{m}$ - and $20 \mu \mathrm{m}$-size spot respectively. Figure 7 shows $10 \times 10$ square patterns drawn and grafted on glass substrate with micrometer-size high resolution. The optical profile of the hydrogel enclosures is obtained with the optical mire exposed to the laser spot. The optimal conditions of UV irradiation are determined as function of the laser power and the writing speed. The calibration is performed by using the optical mire of one hundred squares, each square being drawn by a unique crossing of the laser spot. For the lines of the mire, the laser power varies with a constant writing speed. For the columns of the mire, the writing speed varies with the same laser power. The square enclosures exposed to very weak irradiation (on the right upper 
corner) are eliminated due to partial cross-linking, the uncross-linked polymer chains being removed by rinsing. The square enclosures which are not sufficiently irradiated are a little ondulated due to the wrinkling of weakly cross-linked hydrogels. From the optical profilometer image, the thickness of hydrogel film of each square is deduced. Calibration cartography can be then determined with the hydrogel thickness as function of the parameters of the laser irradiation, the writing speed and the laser power as shown in Figure 7B for the laser spot of $20 \mu \mathrm{m}$ diameter. The UV energy received by the hydrogel patterns for the cross-linking is proportional to $\frac{P}{\phi \times v}$ where $P$ is the laser power, $\phi$ the spot diameter and $v$ the writing speed. For the same laser power, the energy only depends on the product of the spot diameter and the writing speed so that the writing speed is ten times higher for the $2 \mu \mathrm{m}$-size laser spot than for the $20 \mu \mathrm{m}$-size laser spot. Calibration cartography can also be represented by the thickness of the hydrogel film as function of the UV energy received by the cross-linked patterns as shown in Figure 7C. The kinetics of polymer cross-linking through thiol-ene reaction using the $50 \mathrm{mWatt}$ laser is the same as obtained with the common 8 Watt fluorescent lamp. The thickness of the polymer network increases and reaches its maximum. For lower energies, the increase of the film thickness is due to partial cross-linking, the uncross-linked chains being removed by washing. It means that the synthesis of surface-attached of hydrogel patterns using the 50 mWatt laser technology must be performed with UV energy (in arbitrary unit equal to $\frac{P}{\phi \times v}$ ) comprised between 200 and 600 .

Figure 8 displays square enclosures patterns of hydrogels grafted on glass substrate using $20 \mu \mathrm{m}-$ size and $2 \mu \mathrm{m}$-size laser spot. The images of the height profiles are shown in artificial colors. The glass slide is colorized in blue while the hydrogel patterns are green to red, the colors depending on the local thickness. 140 microns-wide squares with 25 microns-wide and 3.5 
microns-thick walls are obtained using $20 \mu \mathrm{m}$-size spot while 70 microns-wide squares with 5 microns-wide and 3.5 microns-thick walls can be obtained with $2 \mu \mathrm{m}$-size laser spot. The height profiles demonstrate that surface-attached hydrogel patterns can be obtained with various resolutions depending on the laser spot size and in particular micrometer-size patterns can be drawn. It should also be highlighted that in addition to allowing the fabrication of micrometersize patterns, the laser lithography method has the time-saving advantages. For example, 10x10 square patterns as those shown in Figures 7 and 8 can be drawn in ten minutes.

A

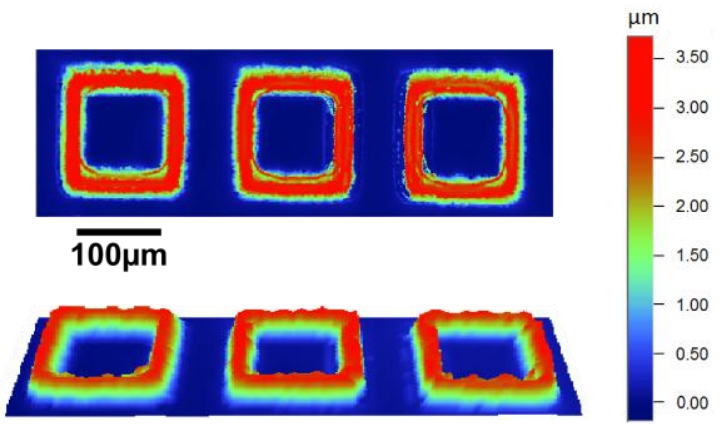

B

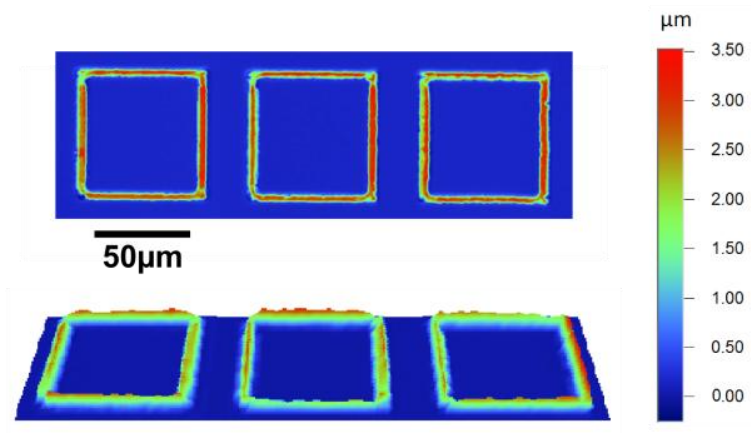

Figure 8. (A) Top view and $3 D$ view of patterned PNIPAM hydrogel squares on glass substrate using the laser spot of $20 \mu \mathrm{m}$. Height profiles are acquired with an optical profilometer. (B) Top view and $3 D$ view of PNIPAM hydrogel squares grafted on glass substrate using the laser spot of $2 \mu m$.

As proved in Figure 9, this versatile and simple method for the fabrication of surface-attached hydrogel patterns also enables the development of multiscale stable hydrogel patterns with multi- 
responsive properties. Surface-attached hydrogel patterns with various shapes and sizes can be easily fabricated. The patterns can be made of various stimuli-responsive polymer networks, the combination being obtained in the internal structure of the layers or distributed in the substrate plane. The submicrometer-thick patterns grafted on reflective silicon wafer show visible colors. The colors are based on interferences theory or Newton's interference colors. It depends on the optical pathlength, or the product of the refractive index and the thickness of the polymer layer and the observation angle.

Figure 9 shows top view pictures of hydrogel pyramidal structure of millimeter size grafted on silicon wafer. The pictures are taken with a general use camera. The pyramidal structure is made of four hydrogel layers and contains two different responsive hydrogels, PNIPAM hydrogel which responds to temperature and PAA hydrogel which responds to $\mathrm{pH}$. The pyramid is fabricated using 8 Watt UV lamp with photomask. It is obtained by alternatively coating enefunctionalized PNIPAM and PAA layers, each layer being successively irradiated (at $254 \mathrm{~nm}$ ) through suitable photomask.

Optical microscopy image of micro-patterned Olympic rings made of surface-attached PNIPAM hydrogels is also displayed. The Olympic rings patterns with high resolution are obtained here using DILASE laser lithography by direct drawing without photomask. The Olympic rings are achieved with PNIPAM hydrogels of various thickness, which provides the color to the hydrogel layer. Ene-functionalized PNIPAM is coated with the suitable thickness and is cross-linked under laser irradiation with $2 \mu \mathrm{m}$-size spot, the uncross-linked chains being removed by washing. The Olympic rings are obtained by five successive coatings / UV irradiation / washing. The correspondence between the rings color and the hydrogel thickness is in good agreement with Newton's interference colors. 

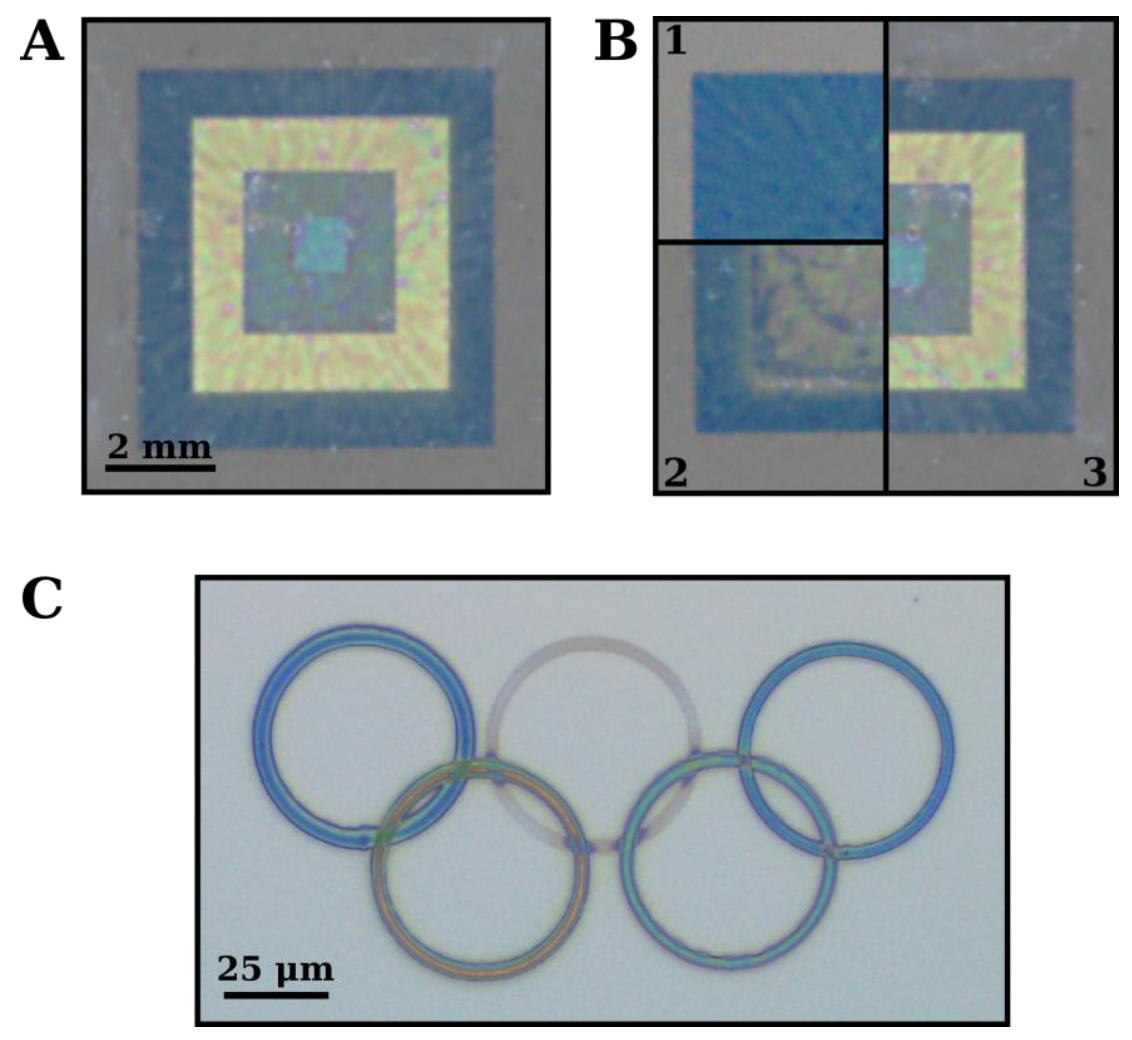

Figure 9. (A) Top view picture of hydrogel patterns with pyramidal multilayers structure covalently grafted onto silicon wafer. The picture is taken with a general use camera Nikon D7000. The pyramidal structure is achieved by alternating PNIPAM and PAA hydrogel layers, each layer being coated and irradiated with common 8 Watt lamp at $254 \mathrm{~nm}$ through suitable photomasks. (B) Image with the different steps for the formation of the pyramidal structure: the blue square base is obtained by coating 140 nm-thick layer from 2 wt\% PNIPAM solution (1), the yellow square corresponds to PAA hydrogel on top of the former PNIPAM hydrogel with a total thickness of $280 \mathrm{~nm}$, the $140 \mathrm{~nm}$-thick PAA layer being obtained from $2 \mathrm{wt} \%$ solution (2), the next two hydrogel layers are 140 nm-thick PNIPAM (2 wt\%) and PAA (2 wt\%) layers (3). (C) Optical microscopy image of hydrogel patterns with Olympic rings shape. The Olympic rings are made of surface-attached PNIPAM hydrogels. These rings patterns with high resolution are 
achieved using DILASE laser lithography by direct drawing without photomask. The Olympic rings are obtained by five successive coatings / UV irradiation / washing. From left to right, the first ring corresponds to a thickness of $240 \mathrm{~nm}$ by coating a $2 \mathrm{wt} \%$ solution at $1000 \mathrm{rpm}$, second ring: $110 \mathrm{~nm}$ with the same $2 \mathrm{wt} \%$ solution at $4000 \mathrm{rpm}$, third ring: $80 \mathrm{~nm}$ with $8000 \mathrm{rpm}$, fourth ring: $220 \mathrm{~nm}$ with $4000 \mathrm{rpm}$ and fifth ring: $140 \mathrm{~nm}$ with $2000 \mathrm{rpm}$. A calibration with the spincoating (concentration of PNIPAM solution and spinning speed) and the laser writing (laser power and writing speed) conditions, is shown in the Supporting Information.

Figure 10 shows fluorescence microscopy images of microfluidic channels filled with fluorescein solution. PNIPAM hydrogel star pattern (with dry thickness of $1.5 \mu \mathrm{m}$ ) is covalently attached to the bottom wall of the ( $8 \mu \mathrm{m}$-high) microchannel. Out of the hydrogel star pattern, the fluorescence intensity is the same bright as it only depends on the height of the channel. At the place where the hydrogel star pattern is surface-attached, the fluorescence intensity depends on the free height of the channel. At temperatures below the LCST (for example $25^{\circ} \mathrm{C}$ ), the star hydrogel is swollen and then appears dark as the fluorescence intensity at this place is weak. For temperatures above the LCST (for example $40^{\circ} \mathrm{C}$ ), the hydrogel pattern is collapsed and then appears bright as the free height of the channel is larger. The free height of the channel and consequently the thickness of the hydrogel in the channel can be deduced from the fluorescence intensity. The calibration of the intensity of the fluorescence signal in grayscale was achieved using a stairway channel with five different heights. The fluorescence intensity was measured for each stair for temperatures ranging from 20 to $50^{\circ} \mathrm{C}$. It was found to be the same for each stair (with a maximum deviation of $5 \%$ from the mean value) confirming no dependence with 
temperature. The normalized fluorescence intensity (to the maximum value measured for the highest stair channel) increases linearly with the channel height as expected; the calibration of fluorescence intensity is displayed on the microscopy image of the stairway channel in Figure 10. The thickness of the hydrogel stair pattern (and consequently the swelling ratio) in the channel at various temperatures can be then determined from the measure of the fluorescence intensity. For temperatures below the LCST $\left(<32^{\circ} \mathrm{C}\right)$, the PNIPAM hydrogel is supposed to be totally swollen. The thickness of the star pattern in water is found to be about four times the dry thickness measured in air before the integration into the microfluidic channel, which is in good agreement with the value determined by ellipsometry and shown in Figure 4. For temperatures above the LCST $\left(>32^{\circ} \mathrm{C}\right)$ with the PNIPAM hydrogel supposed to be collapsed, the swelling ratio found is around 1.5, again in good agreement with ellipsometry measurements.

The hydrogel swelling is homogeneous on the whole star pattern. The star pattern has exactly the same shape in air and in water with no lateral swelling or edges deformation. Actually, as the hydrogel pattern is synthesized in dry state in air and then exposed to water, the hydrogel buckling would arise from inhomogeneous compressive strains due to surface attachment. These buckling instabilities were observed for surface-attached hydrogel patterns for which the thickness and the width are the same order of magnitude. ${ }^{25}$ In our case, the surface-attached hydrogel pattern is much wider than thick (the thickness is a few micrometers while the star size is close to millimeter) so that the swelling of hydrogel is unidirectional (perpendicular to the substrate) without lateral deformation. 

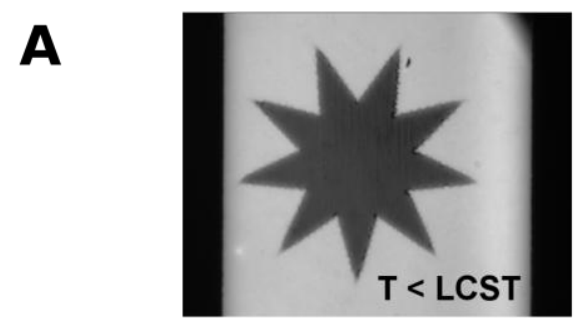

$200 \mu \mathrm{m}$
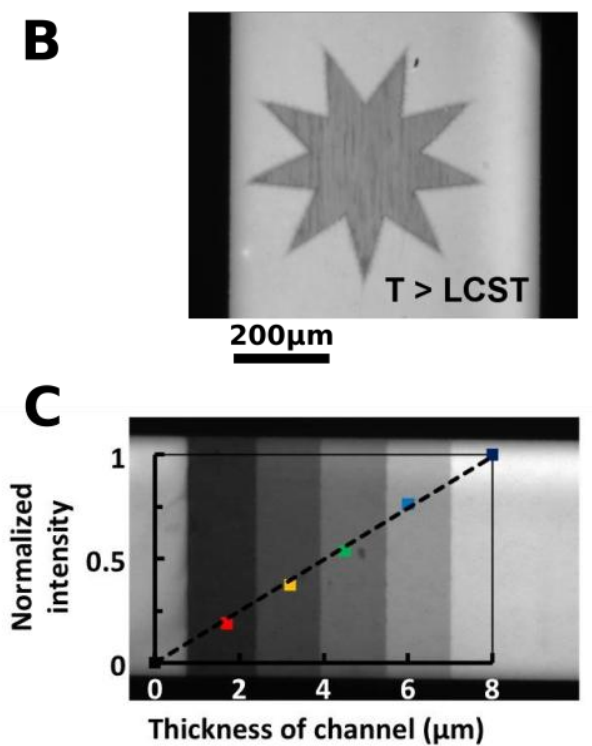

fluorescence

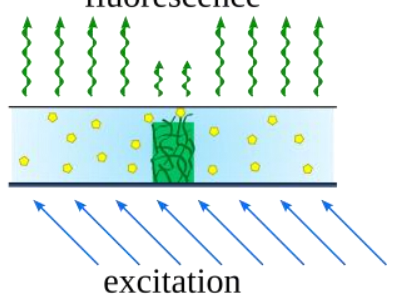

excitation

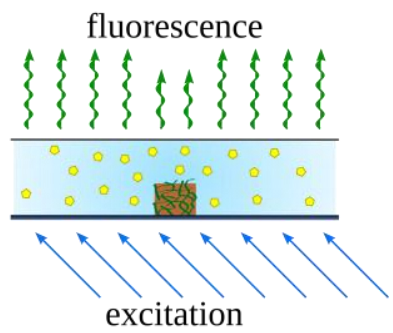

excitation

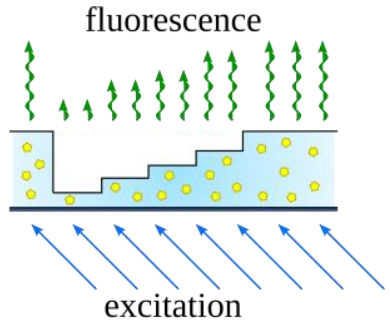

Figure 10. Fluorescence microscopy images in a microfluidic channel. (A) and (B) Pictures of a star pattern of PNIPAM hydrogel covalently grafted on the bottom wall of the microchannel, the dry thickness of the hydrogel being $1.5 \mu \mathrm{m}$ and the height of the channel $8 \mu \mathrm{m}$. The hydrogel pattern which is swollen below the LCST appears dark (A) and when it is collapsed above the LCST, it appears bright (B). On the right, schematic of fluorescence intensity through a microchannel filled with fluorescein solution. At a place where the hydrogel pattern is surfaceattached, the fluorescence intensity depends on the free height of the channel. It is weak for swollen hydrogel and high for collapsed hydrogel. (C) Fluorescence picture and schematic of a stairway microchannel with the representation of the variation of the normalized intensity as function of the channel thickness. 


\section{CONCLUSIONS}

A facile and versatile method for the patterning of surface-attached hydrogels by photolithography was demonstrated. It consisted in cross-linking and surface-grafting preformed ene-reactive polymers through thiol-ene click chemistry. Photolithography was performed with photomasks using common UV lamp and also without photomasks by direct drawing using laser technology. The main characterization of the surface-attached hydrogels was the measure of the thickness of the surface-attached hydrogels in the dry state (in air) and in the swollen state (in water). The systematic characterization of the thickness enabled the control and optimization of the synthesis. We demonstrated the fabrication of micrometer-resolute patterned hydrogels with multi-responsive properties and multiple architectures. In addition to the control of size and shape of hydrogel patterns, the internal layered structure of the patterns can be tailored with any

responsive polymer and with any thickness ranging from a few nanometers to several micrometers. This versatile approach to multi-responsive patterned hydrogels should find wide applications as smart actuators, sensors as well as scaffolds for tissue engineering or substrates for cell culture.

\section{ACKNOWLEDGMENT}

We gratefully thank the French National Research Agency (ANR), the China Scholarship Council (CSC) and the Ministry of Science and Technology of Thailand (MOST) for their financial support. 


\section{REFERENCES.}

1. Nikkhah, M.; Edalat, F.; Manoucheri, S.; Khademhosseini, A. Engineering Microscale Topographies to Control the Cell-Substrate Interface. Biomaterials 2012, 33, 5230-5246.

2. Beebe, D. J.; Moore, J. S.; Bauer, J. M.; Yu, Q.; Liu, R. H.; Devadoss, C.; Jo, B. H. Functional Hydrogel Structures for Autonomous Flow Control Inside Microfluidic Channels Nature 2000, 404, 588-590.

3. Yu, C.; Mutlu, S.; Selvaganapathy, P.; Mastrangelo, C. H.; Svec, F.; Fréchet, J. M. Flow Control Valves for Analytical Microfluidic Chips Without Mechanical Parts Based on Thermally Responsive Monolithic Polymers. Anal. Chem. 2003, 75, 1958-1961.

4. Richtering, W.; Saunders, B. R. Gel Architectures and their Complexity. Soft Matter 2014, $10,3695-3702$.

5. Döring, A.; Birbaum, W.; Kuckling, D. Responsive Hydrogels - Structurally and Dimensionally Optimized Smart Frameworks for Applications in Catalysis, Micro-System Technology and Material Science. Chem. Soc. Rev. 2013, 42, 7391-7420.

6. Cohen Stuart, M. A.; Huck, W. T. S.; Genzer, J.; Müller, M.; Ober, C.; Stamm, M.; Sukhorukov, G. B.; Szleifer, I.; Tsuruk, V. V.; Urban, M.; Winnik, F.; Zauscher, W.; Luzinov, I.; Minko, S. Emerging Applications of Stimuli-Responsive Polymer Materials. Nature Mater. 2010, 9, 101-113.

7. Halperin, A.; Kröger, M.; Winnik, F. M. Poly( $N$-isopropylacrylamide) Phase Diagrams: Fifty Years of Research. Angew. Chem. Int. Ed. 2015, 54, 15342-15367.

8. Li, M.; Bresson, B.; Cousin, F.; Fretigny, C.; Tran, Y. Submicrometric Films of SurfaceAttached Polymer Network with Temperature-Responsive Properties. Langmuir 2015, 31, 11516-11524. 
9. Chollet, B.; Li, M.; Martwong, E.; Bresson, B.; Fretigny, C.; Tabeling, P.; Tran, Y. Multiscale Surface-Attached Hydrogel Thin Films with Tailored Architecture. ACS Applied Materials and Interfaces 2016, 8, 11729-11738.

10. Tokarev, I.; Minko, S. Stimuli-Responsive Hydrogel Thin Films. Soft Matter 2009, 5, 511524.

11. Vidyasagar, A.; Majewski, J.; Toomey, R. Temperature Induced Volume-Phase Transitions in Surface-Tethered Poly(N-Isoprylacrylamide) Networks. Macromolecules 2008, 41, 919924.

12. Toomey, R.; Freidank, D.; Rühe, J. Swelling Behavior of Thin, Surface-Attached Polymer Networks. Macromolecules 2004, 37, 882-887.

13. Harmon, M. E.; Kuckling, D.; Franck, C. W. Photo-Crosslinkable PNIPAAm Copolymers. 2. Effects of Constraint on Temperature and $\mathrm{pH}$-Responsive Hydrogel Layers. Macromolecules 2003, 36, 162-172.

14. Harmon, M. E.; Kuckling, D.; Pareek, P.; Franck, C. W. Photo-Crosslinkable PNIPAAm Copolymers. 4. Effects of Copolymerization in Cross-Linking on the Volume-Phase Transition in Constrained Hydrogel Layers. Langmuir 2003, 19, 10947-10956.

15. Koh, W. G.; Itle, L. J.; Pishko, M. V. Molding of Hydrogel Microstructures to Create Multiphenotype Cell Microarrays Anal. Chem. 2003, 75, 5783-5789.

16. Moeller, H. C.; Mian, M. K.; Shrivastava, S.; Chung, B. G.; Khademhosseini A. A Microwell Array System for Stem Cell Culture. Biomaterials 2008, 29, 752-763.

17. Kobel, S.; Limacher, M.; Gobaa, S.; Laroche, T.; Lutolf, M. P. Micropatterning of Hydrogels by Soft Embossing. Langmuir 2009, 25, 8774-8779. 
18. Revzin, A.; Russell, R. J.; Yadavalli, V. K.; Koh, W. G.; Deister, C.; Hile, D. D.; Mellott, M. B.; Pishko, M. V. Fabrication of Poly(Ethylene Glycol) Hydrogel Microstructures using Photolithography. Langmuir 2001, 17, 5440-5447.

19. Schmaljohann, D.; Nitschke, M.; Schulze, R.; Eing, A.; Werner, C.; Eichhorn, K. J. In situ Study of the Thermoresponsive Behavior of Micropatterned Hydrogel Films by Imaging Ellipsometry Langmuir 2005, 21, 2317-2322.

20. Chen, G.; Kawazoe, N.; Fan, Y.; Ito, Y.; Tateishi, T. Grid Pattern of Nanothick Microgel Network. Langmuir 2007, 23, 5864-5867.

21. Szilagyi, A; Sumaru, K.; Sugiura, S.; Takagi, T.; Shinbo, T.; Zrinyi, M.; Kanamori, T. Rewritable Microrelief Formation on Photoresponsive Hydrogel Layers. Chem. Mat. 2007, 19, 2730-2732.

22 Bartolo, D.; Degré, G.; Nghe, P.; Studer, V. Microfluidic Stickers. Lab Chip 2008, 8, 274279.

23. Yim, H.; Kent, M. S.; Mendez, S.; Balamuragan S. S.; Balamuragan S.; Lopez, G. P.; Satija, S. Temperature-Dependent Conformational Change of PNIPAM Grafted Chains at High Surface Density in Water. Macromolecules 2004, 37, 1994-1997.

24. Yim, H.; Kent, M. S.; Satija, S.; Mendez, S.; Lopez, G. P.; Satija, S.; Seo, Y. Effects of Grafting Density and Molecular Weight on the Temperature-Dependent Conformational Change of Poly(N-Isopropylcrylamide) Grafted Chains in Water. Macromolecules 2006, $39,3420-3426$.

25. DuPont Jr, S. J.; Cates, R. S.; Stroot, P. G.; Toomey, R. Swelling-Induced Instabilities in Microscale, Surface-Confined Poly(N-Isopropylacrylamide) Hydrogels. Soft Matter 2010, 6, 3876-3882. 
TABLE OF CONTENTS

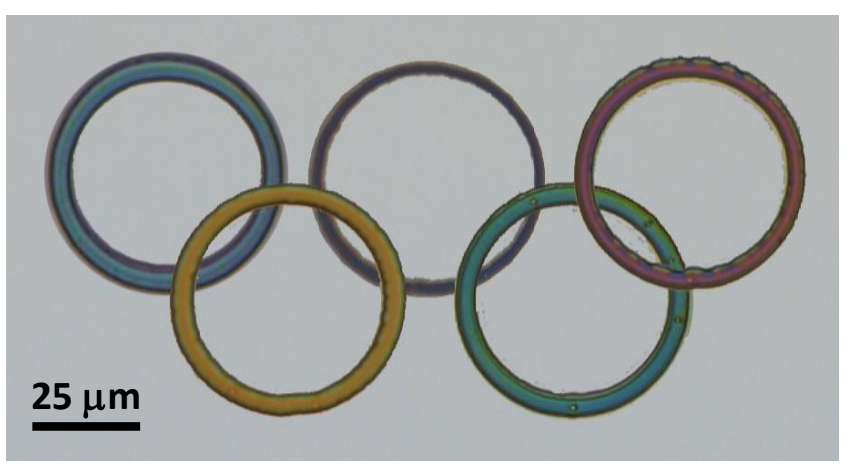

